\title{
Interactive Machines and Its Implication in Scientific Realism
}

\begin{abstract}
Shuai Han
The McCallie School, Chattanooga, Tennessee, USA, 37404,

DavidHan22@mccallie.org

ABSTRACT

Since the tech boom in the early 2000s, computer science, especially the study of algorithms and mathematics, has been prevalent to satisfy new needs for efficiency and convenience. The founding father of modern computer science, Alan Turing, proposes the foundation of modern computability theories known as the Turing Machine. This article is dedicated to exploring a variant of Turing's mathematical model, where the inputs become infinite and empirical. In a way, it is also an exploration of whether theoretically a true interactive machine can be achieved through a finite number of noninteractive machines. To prove it possible, this article utilizes methods such as researching from established papers, proof by deduction, and mathematical induction. In terms of articles, the main source is JSTOR. As the proof goes, it is mathematically proven in this essay that in terms of generic case $K$, an Interactive Machine $A(K)$ shares the same explanatory equivalence with a finite number of Turing Machines $\mathrm{T}(\mathrm{K})$. It is said that A maps onto T.
\end{abstract}

Keywords: Computer Science, Interactive Machine, Scientific Realism, Philosophy, Logic

\section{INTRODUCTION}

As Alonzo Church coined the term "Turing Machine" 1937 when reviewing his paper on computational devices for real numbers, the field of computability and theoretical computer science would be never the same again, essentially establishing a basis for the later research of Interactive Machines, ultimately the study of Empirical Computer Science. Defining expressiveness of systems by observable behavior rather than by inner system-transformation power captures the essence of empirical computer [1]. Scientific Realism, a term often associated with research about its opposite concept, Idealism. Essentially, the description of scientific realism as a positive epistemic attitude toward theories. Overall, Scientific Realism mainly deals with three dimensions, Metaphysic, Semantics, and Epistemology.

This essay mainly deals with Epistemology. When forming an empirical prediction for a scientific theory, the prediction $\mathrm{n}$ is mainly based on knowledge and intuition. In the traditional Turing Machine, it seems absurd to find any intuitions in the strict logical system, whereas the Interactive Machine is solely based on intuition to perform. Applying empirical principles such as observation and inferences, this new field of study effectively provides possibilities for reevaluating the quality of Scientific Realism. To explore such implications on Scientific Realism, a proper introduction to logics that have to deal with understanding specific concepts in the field of computer science, especially, Computability. With the helps computability theories for Interactive Machines, to define expressiveness of systems by observable behavior rather than by inner system-transformation power captures that Machine Learning and Artificial Intelligence can be realized solely by the traditional non-interactive system.

In this essay, the papers from Gödel, Turing, Church, and Wagner are going to be the main source for exploring the logic. To prove specific qualities of Interactive Machines, mathematical induction and deduction are utilized.

\section{UNDERSTANDING INTERACTIVE MACHINES}

\subsection{Turing Machine}

A Turing Machine is a mathematical model that stores data on a strip of tapes. Given a certain function, the input can be computed to meet the result. Given any computer algorithms, there exists a Turing Machine constructed to simulate the algorithm's logic [2].

Attached Figure 1 is a complete configuration of some generic Turing Machine K: 


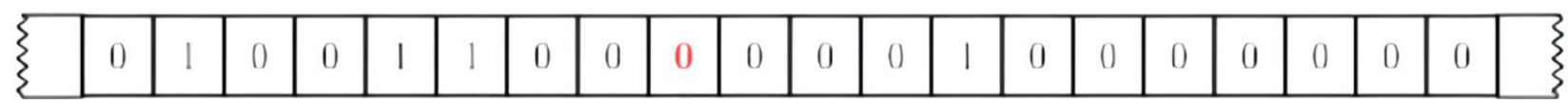

Figure 1 A visualization of Turing Machine

\subsection{Interactive Machine}

An Interaction Machine is Turing Machine extended with input and output actions having the interaction property. Fundamentally, Interactive Machines have input and output actions that interact with an external environment not under its control known as the "Interactive Property" [3]. To be more specific, Interactive Machines themselves accept infinite amounts.
An interactive machine can be abductively considered as two parts, a public observer, $\mathrm{O}_{\mathrm{p}}$, which actively captures inputs from outside the system; and a hidden noninteractive system (similar to Turing Machine), $\mathrm{O}_{\mathrm{T}}$. In real life, an application for interactive machines can be seen in the field of Artificial Intelligence. However, the realization of a truly interactive and autonomous machine seems to be in doubt.

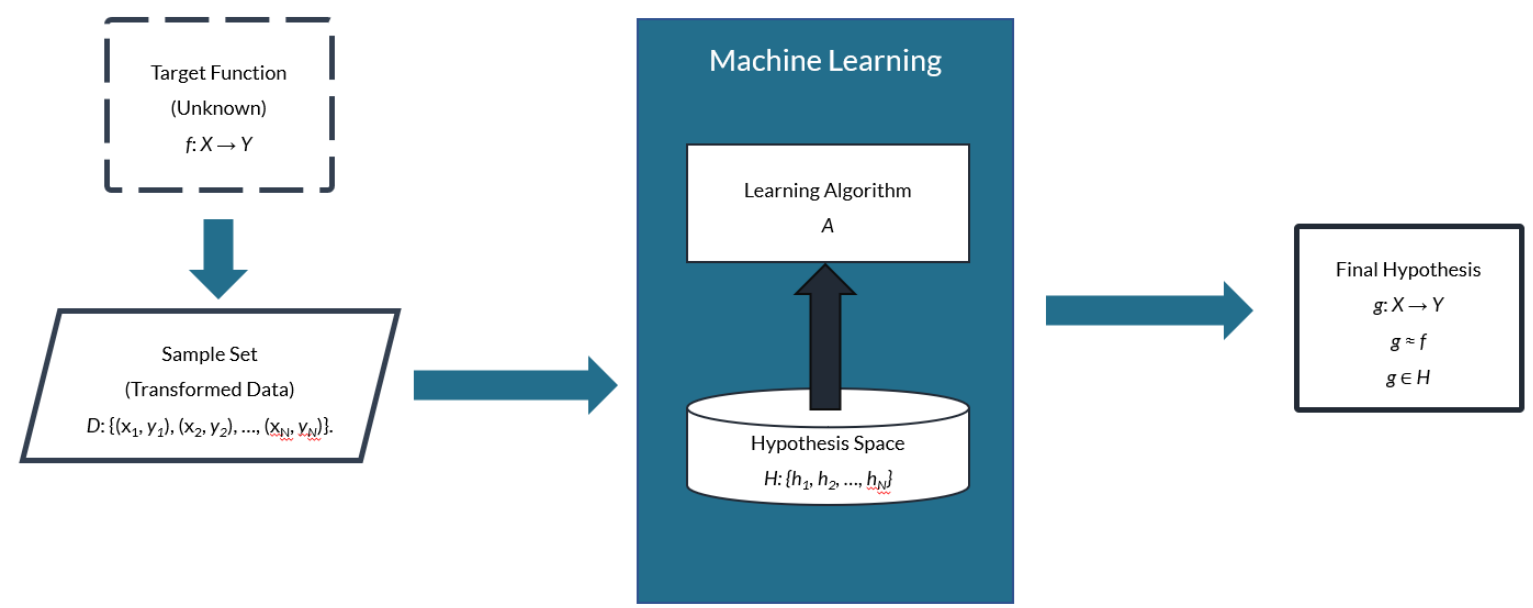

Figure 2 A visualization of Interactive Machine in Machine Learning

\section{PROPERTIES OF INTERACTIVE MACHINES}

\subsection{Algorithms in Empirical Computer Science}

As Turing - Church Thesis states that a function on the natural numbers can be calculated by an effective method if and only if it is computable by a Turing machine. This implies Turing Machines take a finite set of noninteractive inputs and then transform them into outputs. It helps to define Algorithms on the basis of modern computer science, a computer-implementable function that takes a finite set of real numbers then mapping onto its results. To better analyze the efficiency of algorithms, the term of time complexity is denoted $\mathrm{O}(\mathrm{n})$ and space complexity is also $\mathrm{O}(\mathrm{n})$ asymptotically, where $\mathrm{n}$ in both concepts is the finite numbers of input. Yet, When the concept of computability changes from noninteractive Turing Machines to Interactive Machines that accept infinite sets of inputs through observations, a more complex set of algorithms is needed to solve new programs. As a matter of fact, the study of complex algorithms can also be conducted empirically. The field, empirical algorithms, indicate that the evaluation of an algorithm's efficiency can be done using statistics [4]. By comparing the difference between predictions and actual through experimentation, an interactive machine is strictly qualified to be a scientific model (Hume).

\subsection{Logic in Interactive Machines}

Further, into the realm of Empirical Computer Science, the notion of logic also changes. In mathematical logic, as Kurt Gödel's Incompleteness theorem says that suppose there is a logical system called $\mathrm{K}$ [5]. That is, $\mathrm{K}$ is incomplete if and only there exists a true proposition assertion $\mathrm{X}$ and its negation $-\mathrm{X}$ that are not theorems in $\mathrm{K}$.

\subsubsection{Prove that Interactive Machines are Incomplete:}

Suppose there is a Logical System K, K contains all theorems from Interactive Machines. In a program $\mathrm{P}$ with Interactive Machines: Given the infinite input as $\mathrm{x}$, $\left(\exists_{\mathrm{x}}\right)$ (Input $\left.(\mathrm{x}): \mathrm{x} \in \mathrm{R}\right)$ then using the algorithm $\mathrm{A}_{1}$ in transforming inputs to a formal statement $S_{x}(n)$, then using a second algorithm $A_{2}$ to determine whether $S_{x}(n)$ is a theorem of $\mathrm{K}$. Therefore, $\mathrm{S}_{\mathrm{x}(\mathrm{n})} \leftrightarrow \mathrm{P}$.

According to the description, the Program is false if and only if $S_{x}(n)$ is not a theorem of $K$. 
Proved by Arthur Charlesworth, there exists a predicate for the program that there is $S_{p}(n)$ that program is false if and only if $S_{p}(n)$ is a theorem of $K$. (Charlesworth 1981) Since the inputs for interactive machines are infinite and are elements of the set of all real numbers. Then, a paradox is made, $S_{p}(n)$ is a not theorem of $\mathrm{K}$, and $\mathrm{S}_{\mathrm{p}}(\mathrm{n})$ is a theorem of $\mathrm{K}$.

Hence, Interactive Machines are incomplete.

Such incompleteness result shows a limitation of rationalism mathematics. It certainly shows that neither the logic of mathematics or Turing Machines (Noninteractive Logic) cannot reduce to Interactive Machines' Logic. Also, it is important to discover the soundness of Interactive Machines. According to the definition, a logical system is considered sound if and only if when all the theorems in the system are tautologies. When considering the case of an argument, an argument is sound when it is valid and premises are true.

\subsubsection{Prove that Interactive Machines are Sound:}

A system of logic is sound if all of its theorems are true statements; a system of logic is complete if all true assertions of the domain are theorems [6]. Studies of sound and logic help better understand the relationship between Empirical Computer Science and Logic. As Church in his thesis mentions, every algorithm corresponds to a recursive function [7]. It implies a sense of correspondence within inner realities.

\section{SCIENTIFIC REALISM IN INTERACTIVE MACHINES}

Under the notion of logic, a rule of correspondence is also implied, 'for interactive systems, properties correspond to observations and the number of properties corresponds to the number of distinct observations.[6]. Yet, the notions about observations and properties are reserved without further elaboration.

In Scientific Realism, there are two parts of the world, the physical world, where human observations happen; and the mind world, where lies beyond the senses. As Kant points out that, space and time are forms of intuition that then structures all observations [8].

In the design of Interactive Machines, there are two parts of the system, interactive port, which constantly takes real-number inputs; and noninteractive port, through algorithms. As Church points out in his paper that as long as it is can be physically computed, a device can be simulated using Turing Machine [9]. Noted that Church is directly referring to the noninteractive port --recursive functions to be more specific --- about its limitations in the physical world. Yet, when the notion changes to Interactive Machines, the notion of physical seems to no longer work perfectly in that sense.

Yet, in the process of getting data alone, seems to be possible to be considered by another Turing Machine. In this section, to prove the point that Interactive Machines are essentially two parts of the Turing Machine, a tool of Explanatory Power and Computational Expressiveness is needed.

Definition of Explanatory Power is the notion of Explanator Power is formally understood by associating a theory with observation. It is measured by the correspondence between the prediction made by theory with the actual observation[9]. Definition of Expressiveness is the Expressiveness of an observer's ability to make an observational distinction [9].

First, assume there is an Interactive Machine, $\mathrm{I}_{\mathrm{T}}$, the primary observer of Interactive Machine is $\mathrm{O}_{t}$, and the hidden interface is $T_{I}$. To prove that $O_{t}$ and $T_{I}$ are equivalent, there is a need to prove that their explanatory power is the same, meaning that they have the capability to correspond the same between prediction and theory.

Suppose the setting a park, when an interactive machine is activated to take an observation in about Objects in the park, the input is a set consisted by real numbers denoted $\mathrm{K}=\{\mathrm{a}: \mathrm{a} \in \mathrm{Q}\}$ since the basic programming is in binary mode. Then, the set $\mathrm{K}$ is processed through Primary Observer, $\mathrm{O}_{\mathrm{t}}$, then prints the output, a scientific theory $\mathrm{T}_{\mathrm{im}}$.

In Turing Machines, the primary observer would be considered as such:

$$
\frac{1}{n} \sum_{n+1}^{n+n^{\prime}} L\left(h\left(x^{i}\right), y^{i}\right)
$$

Where, $\mathrm{L}(\mathrm{x}, \mathrm{y})$ stands for the loss function, $\mathrm{x}$ indicates prediction function, and $\mathrm{y}$ is actual result.

\subsection{Case 1: classifying an object}

For Turing Machine, the equation would be:

$$
\begin{array}{r}
g=h\left(x ; \theta_{1}, \theta_{0}\right)=\operatorname{sign}\left(\theta_{x}^{T}+\theta_{0}\right)=\left\{\begin{array}{c}
+1 \\
-1
\end{array}\right. \\
x \in R^{D}, \text { for } D \in Z \\
L(g, a)=\left\{\begin{array}{l}
0 \\
1
\end{array}\right.
\end{array}
$$

Where, $g$ stands for the predicted result, and a is the actual result. $\mathrm{L}(\mathrm{g}, \mathrm{a})$ stands for the explanatory power function.

For Interactive Machine, the equation would be:

$G(I, O)=\left\{\begin{array}{l}0 \\ 1\end{array}\right.$

Where, I stands for Intuition, O stands for the facts, G $(\mathrm{I}, \mathrm{O})$ stands for the explanatory power function. 


\subsection{Case 2: Regression}

For Turing Machine, the equation would be:

$$
J\left(\theta, \theta_{0}\right)=\frac{1}{n} \sum_{i=1}^{n}\left(\theta^{T} x^{i}+\theta_{0}-y^{i}\right)^{2}
$$

Where the objective is to minimize this equation:

$$
\theta^{*}, \theta_{0}^{*}=\arg \min _{\left(\theta, \theta_{0}\right)} J\left(\theta, \theta_{0}\right)
$$

For interactive Machine, the equation would be:

$$
G(I, O)=g-a
$$

Where $\mathrm{g}$ stands for prediction, and a stand for actual.

Under such circumstances, the explanatory power of each case would be the same: either 0 or 1 . Yet the methods of how things are sorted are different. In the concept of Turing Machine, it is simply a recursive function to evaluate the loss of a linear classifier, the basic capability to predict something, in the result of only Yes or No. In regression, a more complicated function to predict a specific number, an intuition would share the same level of deficiency as Turing Machine, when the number of trials $\mathrm{n}$ is close to infinity. Under this circumstance, the difference between prediction and actual in each case is attributed to the notion of perspective, the difference between observables and unobservable. In real life situation, intuition is to classify a specific observation, and to correspond it with another knowledge is to apply Deductive - nomological Explanation, to apply general laws to explain a phenomenon. This involves two parts, the first part is inductively addressing the observation, and the second is deduced it using logic. Such a notion directly captures the essence of Empirical Computer Science.

In Scientific Realism, it is considered that the physical world exists independently of human consciousness. Intuition, affected by the physical world, is also part of the independent consciousness. As the comparison between Turing Machine and Interactive Machine suggests, intuition cannot stand by itself without the notion of deductive logic. The physical world and Human consciousness are intertwined together in the form of Interactive Machines. The aim of Science by Scientific realism and Empirical Computer Science is not just to provide a true description of Truth, yet also as an instrument to help better progress the study of logic and engineering.

\section{CONCLUSION}

In this essay, it is proved that Interactive Computer is incomplete due to an absurd. Whereas, it is confirmed that Interactive Computer is Sound in the notion that the physical world exists independently of the Mind and Consciousness. To better understand Turing Machine and Interactive Machines in terms of their explanatory equivalence. This essay discusses two generic cases in real-life situations. As indicated in the "classifying an object" case, Turing machines are equivalent to real interactive machines. Whereas, in the case of "regression", it is also the case that Interactive Machines can be represented by Turing Machines. It is proved that Interactive Machines are actually equivalent to a finite number of Turing Machines. Throughout the research, the part of experimentation in empirical science is missing. The theory for proving Turing Machines equivalent to Interactive Machines would be sounder having a set of different scenarios to elaborate the two generic cases. Such understandings help better promote the research into fields such as Machine learning and Artificial Intelligence, wherein turns to help better understand the aim of science and its future.

\section{ACKNOWLEDGMENTS}

First and foremost, I would like to thank Mr. Sumner McCallie for inspiring me to write this essay discussing the potentials of Interactive Machines in real life. Without him, this essay may not be possible.

I cannot express enough appreciation for Ms. Yike Han's help in polishing this essay. There are numerous nuisances in this article, but she managed to make it sound and complete. This essay could be completed without her dedication and attentive care.

Finally, I would like to acknowledge Mrs. Karol Wang's help in helping me find references and articles. Her help has been tremendous and significant in making this essay possible.

\section{REFERENCES}

[1]Kukla, Andre. " The Theory-Observation Distinction.” The Philosophical Review 105, no. 2 (1996): 173 230 https://doi.org/10.2307/2185717.

[2]Turing, Alan, Andrew W. Appel, and Solomon Feferman. Alan Turing's Systems of Logic: The PRINCETON THESIS. Princeton, NJ: Princeton University Press, 2012.

[3]Wegner, Peter " Interactive Foundations of Computing " , Theoretical Computer Science, February (1998)

[4]Wegner, Peter "Why Interaction is More Powerful than Algorithms” , CACM, May 1997.

[5]Gödel, Kurt. “Über Formal Unentscheidbare Sätze Der Principia Mathematica Und Verwandter Systeme I.” Monatshefte für Mathematik. 1931 (November 17, 1930). 
[6]Heijenoort, Jean Van. From Frege to GÖDEL, a Source Book in Mathematical Logic: 1879-1931. Jean Van HEIJENOORT .. Cambridge, UK: Mass., 1967.

[7]De Mol, Liesbeth, "Turing Machines", The Stanford Encyclopedia of Philosophy (Fall 2021 Edition), Edward N. Zalta (ed.), URL = $<$ https://plato.stanford.edu/archives/fall2021/entrie s/turing-machine/>.
[8]Wegner, Peter. "Towards Empirical Computer Science." The Monist 82, no. 1 (1999): 58-108. http://www.jstor.org/stable/27903623.

[9]Charlesworth, Arthur. "A Proof of Godel's Theorem in Terms of Computer Programs." Mathematics Magazine 54, no. 3 (1981): 109-21. https://doi.org/10.2307/2689794 